From the Brain Tumor and NeuroOncology Center and Departments of Neurosurgery and Hematology and Medical Oncology, Cleveland Clinic, Cleveland; Department of Neurosurgery, Ohio State University Medical Center, Columbus, OH; Departments of Neurology, Public Health and Preventive Medicine, Department of Medical Informatics and Clinical Epidemiology, Medicine, and Neurosurgery, Oregon Health \& Science University; Department of Pharmacy Practice, Oregon State University; Portland Veterans Affairs Medical Center, Portland, OR; and Leslie and Michael Gaffin Center for Neuro-Oncology, Hadassah Hebrew University Hospital, Jerusalem, Israel.

Submitted July 29, 2008; accepted January 26, 2009; published online ahead of print at www.jco.org on May 18,2009

Supported by a Veterans Administration Merit Review Grant and National Institutes of Health Grants No. CA137488, NS34608, and NS44687 from the National Cancer Institute and the National Institute of Neurological Disorders and Stroke to E.A.N.

Presented in part at the 43rd Annual Meeting of the American Society of Clinical Oncology, June 3, 2007, Chicago, IL.

Authors' disclosures of potential conflicts of interest and author contributions are found at the end of this article.

Corresponding author: Edward A. Neuwelt, MD, 3181 SW Sam Jackson Park Rd, Mailcode L603, Portland, OR 97239; e-mail: neuwelte@ohsu.edu.

C 2009 by American Society of Clinical Oncology

0732-183X/09/2721-3503/\$20.00

DOI: $10.1200 / J C O .2008 .19 .3789$

\title{
Blood-Brain Barrier Disruption and Intra-Arterial Methotrexate-Based Therapy for Newly Diagnosed Primary CNS Lymphoma: A Multi-Institutional Experience
}

Lilyana Angelov, Nancy D. Doolittle, Dale F. Kraemer, Tali Siegal, Gene H. Barnett, David M. Peereboom, Glen Stevens, John McGregor, Kristoph Jahnke, Cynthia A. Lacy, Nancy A. Hedrick, Edna Shalom, Sandra Ference, Susan Bell, Lisa Sorenson, Rose Marie Tyson, Marianne Haluska, and Edward A. Neuwelt



\section{Purpose}

Primary CNS Iymphoma (PCNSL) is confined to the CNS and/or the eyes at presentation and is usually initially treated with intravenous methotrexate-based chemotherapy and whole-brain radiotherapy (WBRT). However, the intact blood-brain barrier (BBB) can limit diffusion of methotrexate into brain and tumor. With BBB disruption (BBBD), enhanced drug delivery to the tumor can be achieved.

\section{Patients and Methods}

This report summarizes the multi-institutional experience of 149 newly diagnosed (with no prior WBRT) patients with PCNSL treated with osmotic BBBD and intra-arterial (IA) methotrexate at four institutions from 1982 to 2005 . In this series, $47.6 \%$ of patients were age $\geq 60$ years, and $42.3 \%$ had Karnofsky performance score (KPS) less than 70 at diagnosis.

\section{Results}

The overall response rate was $81.9 \%$ (57.8\% complete; $24.2 \%$ partial). Median overall survival (OS) was 3.1 years (25\% estimated survival at 8.5 years). Median progression-free survival (PFS) was 1.8 years, with 5 -year PFS of $31 \%$ and 7 -year PFS of $25 \%$. In low-risk patients (age $<60$ years and KPS $\geq 70$ ), median OS was approximately 14 years, with a plateau after approximately 8 years. Procedures were generally well tolerated; focal seizures $(9.2 \%)$ were the most frequent side effect and lacked long-term sequelae.

\section{Conclusion}

This large series of patients treated over a 23-year period demonstrates that BBBD/IA methotrexate-based chemotherapy results in successful and durable tumor control and outcomes that are comparable or superior to other PCNSL treatment regimens.

\section{J Clin Oncol 27:3503-3509. (c) 2009 by American Society of Clinical Oncology}

\section{INTRODUCTION}

The incidence of primary CNS lymphoma (PCNSL) in immunocompetent patients represents approximately $4 \%$ of intracranial neoplasms. This aggressive, extranodal non-Hodgkin's lymphoma is confined to the CNS and/or the eyes at presentation. Patients age $\geq 60$ years account for more than $50 \%$ of the cases. ${ }^{1}$

Numerous studies suggest high-dose methotrexate $\left(\geq 1 \mathrm{~g} / \mathrm{m}^{2}\right)$, despite its modest blood-brain barrier (BBB) permeability (approximately $5 \%$ of plasma levels), ${ }^{2,3}$ combined with brain irradiation results in improved patient response and prolonged progression-free survival (PFS) and overall survival (OS). ${ }^{4-8}$ Combined-modality therapy, however, is associated with a high incidence of delayed and often devastating neurocognitive toxicity in long-term survivors, especially those older than 60 years. ${ }^{4,9-20}$

We update our previously reported singlecenter results ${ }^{21,22}$ and present our large multiinstitutional experience in the treatment of patients with PCNSL using BBB disruption (BBBD) in conjunction with intra-arterial (IA) methotrexatebased chemotherapy. We report for the first time the PFS in this large series, as well as OS and toxicity. This series is unique in that patients were treated over a 23-year period.

\section{PATIENTS AND METHODS}

\section{Patients}

Newly diagnosed immunocompetent patients with PCNSL $(n=131)$ were enrolled onto the study and 
prospectively treated by the BBBD programs at four institutions (Oregon Health \& Science University, Portland, OR; Cleveland Clinic, Cleveland, $\mathrm{OH}$; Hadassah Hebrew Medical Center, Jerusalem, Israel; and Ohio State University, Columbus, OH) between February 1982 and December 2005. Eligible patients had histologically confirmed PCNSL (by brain biopsy, CSF cytology, or vitrectomy), with no evidence of lymphoma elsewhere in the body and no HIV infection at diagnosis. Before treatment, patients were required to have an absolute granulocyte count more than $1,200 / \mu \mathrm{L}$, platelet count more than $100,000 / \mu \mathrm{L}$, and normal hepatic and renal function. Patients with uncontrolled pulmonary or cardiac complications were not eligible. Uniform protocols were approved by the local institutional review board (IRB) or ethics committee of each institution. Informed consent was obtained from the patients. Newly diagnosed patients (ie, those within 90 days of histologic diagnosis) and those who had not received prior whole-brain radiotherapy (WBRT) are included in this report.

In addition, each institution obtained IRB permission to include newly diagnosed patients with PCNSL $(n=18)$ treated off-protocol with methotrexate-based chemotherapy between the same dates and institutions who met the following criteria: no systemic lymphoma or HIV at diagnosis and no prior WBRT. Therefore, although the vast majority of the patients (131 of $149 ; 88 \%$ ) were enrolled onto a prospective study, results from all patients treated with BBBD at the four institutions between 1982 and 2005 who met the above criteria are included in this report, whether treated on or off protocol.

Pretreatment studies included physical examination, Karnofsky performance score (KPS), cranial magnetic resonance imaging with and without contrast, and extraneural computed tomography staging (chest and abdomen). Before magnetic resonance imaging availability, cranial computed tomography scans were obtained. Bone marrow biopsy and ophthalmologic examination with slit lamp evaluation were performed as indicated, and CSF cytopathology was obtained if lumbar puncture could be safely performed.

\section{Treatment}

Chemotherapy protocols were uniform across the four BBBD consortium institutions. Comprehensive guidelines for anesthesia, transfemoral arterial cannulation, mannitol/chemotherapy infusion, pre- and post-BBBD patient care, and follow-up were centrally developed and followed by trained teams at all institutions.

Osmotic BBBD with IA infusion of methotrexate-based chemotherapy has been described previously. ${ }^{21-24}$ The BBBD and chemotherapy treatment details are summarized in Table 1 . The treatment of intraocular lymphoma included intraocular methotrexate ${ }^{25}$ or ocular radiation. Leucovorin rescue (80 mg intravenously [IV]) was initiated 36 hours after the first dose of methotrexate and continued thereafter for 5 days $(50 \mathrm{mg}$, orally or IV, every 6 hours). ${ }^{21,24,26}$ Patients received two BBBD treatments on 2 consecutive days every 4 weeks for up to 12 monthly courses unless there was evidence of disease progression. ${ }^{21,24}$

\section{Response Evaluation and Follow-Up}

Follow-up studies included complete neurologic examinations, CBCs, ophthalmologic examination, and CSF cytopathology. Neuroimaging studies were obtained before each monthly treatment course, after the final treatment course, and thereafter every 3 months for 1 year, every 6 months for 2 years, and then annually. Standard parameters were used to determine treatment response. ${ }^{27}$ For instance, a complete response (CR) was documented if there was complete resolution of enhancing abnormalities on imaging, no evidence of active ocular lymphoma, negative CSF cytology with absence of leptomeningeal disease-related symptoms, and no corticosteroids.

Standard data collection forms and BBBD chemotherapy records were sent to the BBBD coordinating center (Oregon Health \& Science University) for review and database entry. Data were reviewed for correctness by each institution and centrally. Data was collected through December 2005. Protocol adherence was overseen by the IRB, and quality assurance audits began in 1996 with the establishment of a cancer center at the BBBD coordinating center.

\section{Statistical Methods}

Demographic, baseline, and treatment characteristics were summarized using descriptive statistics. Complications were summarized as both proportion of patients with each complication, the total number of episodes, and the

\begin{tabular}{|c|c|}
\hline Day & Treatment \\
\hline Day 1, admission & $\begin{array}{l}\text { History and physical examination } \\
\text { Routine labs } \\
\text { Neuroimaging }\end{array}$ \\
\hline Days 2 and 3 & $\begin{array}{l}\text { BBBD treatment } \\
\text { General anesthesia } \\
\text { Antiepileptic drugs } \\
\text { Transfemoral catheterization of carotid or } \\
\text { vertebral artery } \\
25 \% \text { warmed mannitol IA infusion over } 30 \\
\text { seconds } \\
\text { IV imaging agent } \\
\text { Chemotherapy* } \\
\text { IA methotrexate } \\
\text { Brain imaging to document disruption }\end{array}$ \\
\hline Day 4 & $\begin{array}{l}\text { Leucovorin rescue } \\
\text { Discharge }\end{array}$ \\
\hline Day 5 & Granulocyte colony-stimulating factor \\
\hline \multicolumn{2}{|c|}{$\begin{array}{l}\text { Abbreviations: BBBD, blood-brain barrier disruption; IA, intra-arterial; } \\
\text { IV, intravenous. } \\
\text { "Between } 1982 \text { and } 1993 \text {, chemotherapy used in combination with } \\
\text { methotrexate included etoposide ( } 150 \mathrm{mg} / \mathrm{m}^{2} \text { IV days } 1 \text { and } 2 \text { ) or cyclo- } \\
\text { phosphamide ( } 15 \mathrm{mg} / \mathrm{kg} \text { IV days } 1 \text { and } 2 \text { ) and procarbazine ( } 100 \mathrm{mg} \text { orally days } \\
3 \text { through } 16 ; 44 \mathrm{patients} \text { ). Between } 1994 \text { and } 2005 \text {, etoposide or etoposide } \\
\text { phosphate ( } 150 \mathrm{mg} / \mathrm{m}^{2} \mathrm{IV} \text { days } 1 \text { and } 2 \text { ) and cyclophosphamide }\left(500 \mathrm{mg} / \mathrm{m}^{2} \mathrm{IV}\right. \\
\text { days } 1 \text { and } 2 \text { ) were used (105 patients). Granulocyte colony-stimulating factor } \\
\text { was added in } 1994 \text {. At that time, etoposide or etoposide phosphate replaced } \\
\text { oral procarbazine. }\end{array}$} \\
\hline
\end{tabular}

rate of episodes per number of procedures. OS and PFS were measured from the first BBBD treatment date to date of first relapse (for PFS), death, or last follow-up. OS and PFS were estimated using Kaplan-Meier estimates. Potential predictors for OS and PFS were assessed by fitting stratified univariate analyses and comparing strata using the log-rank test or the generalized Wilcoxon test (the latter test used when visual comparison of the stratified curves was not consistent with the proportional hazards assumption). Potential predictors of OS and PFS were age ( $<60 v \geq 60$ years), KPS ( $\geq 70 v<70$ ), Memorial Sloan-Kettering Cancer Center (MSKCC) risk classes (class 1, age $<50$ years; class 2 , age $\geq 50$ years and KPS $\geq 70$; class 3 , age $\geq 50$ years and KPS $<70$ ), ${ }^{28}$ sex, CSF cytology (negative, positive, or atypical/suspicious), whether or not the patient discontinued treatment as a result of an adverse event, whether or not the patient had intraocular lymphoma, and whether or not surgical resection was performed. The MSKCC risk scores are effectively a statistical interaction term (the interaction of age and KPS) that uses age more than and less than 50 years. In our previous report, we used age more than and less than 60 years. Both of these age cut-points were assessed in this report. Multivariable Cox proportional hazards models were also fit using these potential predictors. Variable selection was made using a modified forwardvariable selection approach evaluating both changes in the Akaike's information criterion and $P$ values.

\section{RESULTS}

\section{Patient Characteristics}

One hundred forty-nine patients were treated with BBBD followed by IA methotrexate-based chemotherapy (2,079 procedures). Seventy-four of these patients were included in previous reports. ${ }^{21,22}$ Patient characteristics are summarized in Table 2. Seventy-eight women and 71 men were included. The mean age $( \pm S D)$ at diagnosis was 54.5 years ( \pm 15.5 years $) ; 47.6 \%$ were age 60 years or older. Mean 


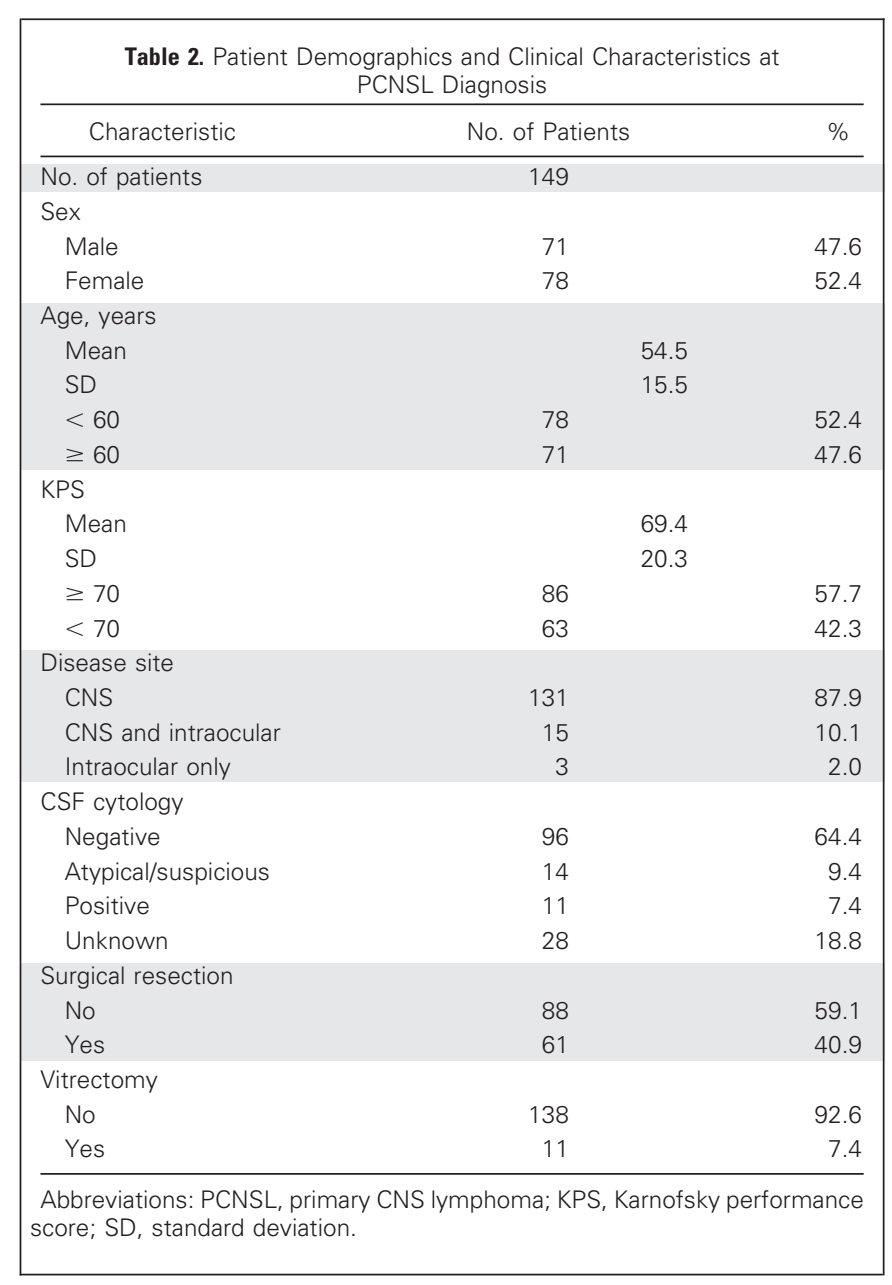

baseline KPS was $69.4( \pm 20.3)$. Sixty-one patients had surgical resection as part of their diagnostic regimen, and 27 patients had started chemotherapy at outside centers and then were referred to a BBB consortium facility for treatment within 90 days of diagnosis. Twentyfive patients had positive CSF for lymphoma cells or atypical cells. The number of patients treated at each center ranged from eight to 104 .

\section{Outcomes}

The 149 patients received a median of 16 IA/BBBD treatments (eight monthly courses). This resulted in clinical and radiographic CR in 86 patients and partial response in 36 patients (overall response rate, $81.9 \%$ ). Stable disease was documented in $8.1 \%$ and progressive disease in $3.4 \%$. Ten patients (6.7\%) were not assessable/unknown. Stratified by age, the response rate was $83.3 \%$ for patients younger than 60 years and $80.3 \%$ for patients $\geq 60$ years. Patients with baseline KPS $\geq 70$ had a response rate of $82.6 \%$ and patients with KPS less than 70 had a response rate of $81.0 \%$.

Fifty-six patients $(37.6 \%)$ had no evidence of disease progression at last follow-up, whereas 93 patients $(62.4 \%)$ experienced disease progression. Median PFS was 1.8 years ( $95 \%$ CI, 1.3 to 2.8 years); 5 -year PFS was $31 \%$ and 7 -year PFS was $25 \%$ (Fig 1). KPS $\geq 70$ $(P=.0007$, Wilcoxon test $)$ and MSKCC risk class $(P<.0001$, Wilcoxon test) were significant predictors of PFS. Age younger than 60 years $(P=.32$, Wilcoxon test $)$, sex ( $P=.64$, Wilcoxon test), CSF

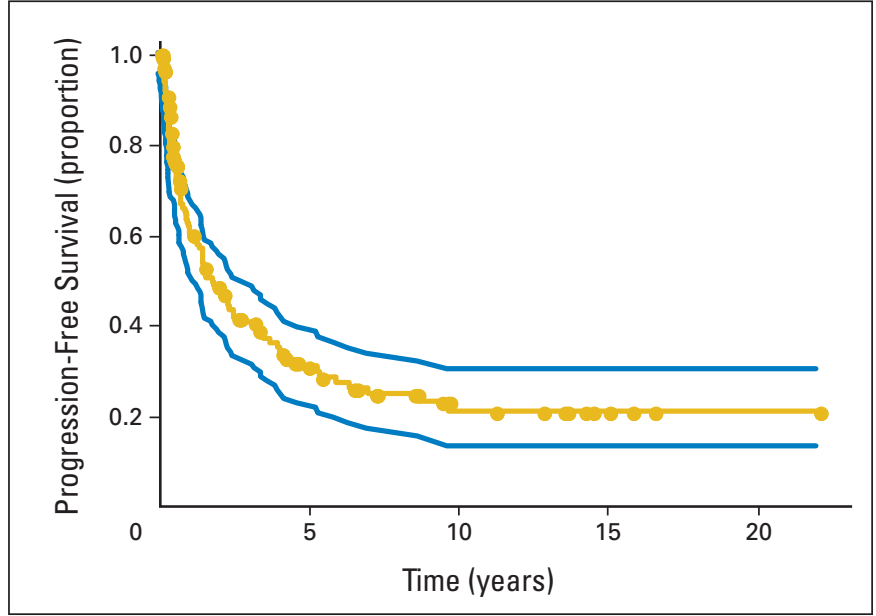

Fig 1. Progression-free survival (yellow line; median, 1.8 years; $95 \% \mathrm{Cl}, 1.3$ to 2.8 years) from date of first intra-arterial/blood-brain barrier disruption treatment (149 patients, 93 have experienced disease progression) with $95 \% \mathrm{Cl}$ (blue lines). Symbols on lines indicate censored observations.

cytology $(P=.28$, Wilcoxon test $)$, discontinuation owing to complications $(P=.24$, Wilcoxon test $)$, intraocular disease $(P=.90$, Wilcoxon test), and surgical resection $(P=.78$, Wilcoxon test) were not significant predictors.

Ninety-three patients experienced relapse, with the longest after 9.7 years. The site of first relapse was CNS only (73 patients, 49.0\%), CNS and intraocular (nine patients, 6.0\%), and intraocular only (two patients, 1.3\%). Nine patients $(6.0 \%)$ experienced relapse systemically.

Ninety-six patients (64.4\%) have died. Median OS was 3.1 years (95\% CI, 2.2 to 5.0 years), with $41 \%$ estimated 5-year survival and 25\% estimated 8.5-year survival (Fig 2). Age younger than 60 years $(P=.0019$, log-rank test $), \mathrm{KPS} \geq 70(P<.0001$, log-rank $)$, MSKCC risk classes $(P<.0001$, log-rank test $)$, and sex $(P=0.039$, Wilcoxon test) were significant predictors for OS. CSF cytology $(P=.93$, Wilcoxon test), discontinuation owing to complications $(P=.92$, Wilcoxon test), intraocular disease $(P=.76$, Wilcoxon test), and surgical

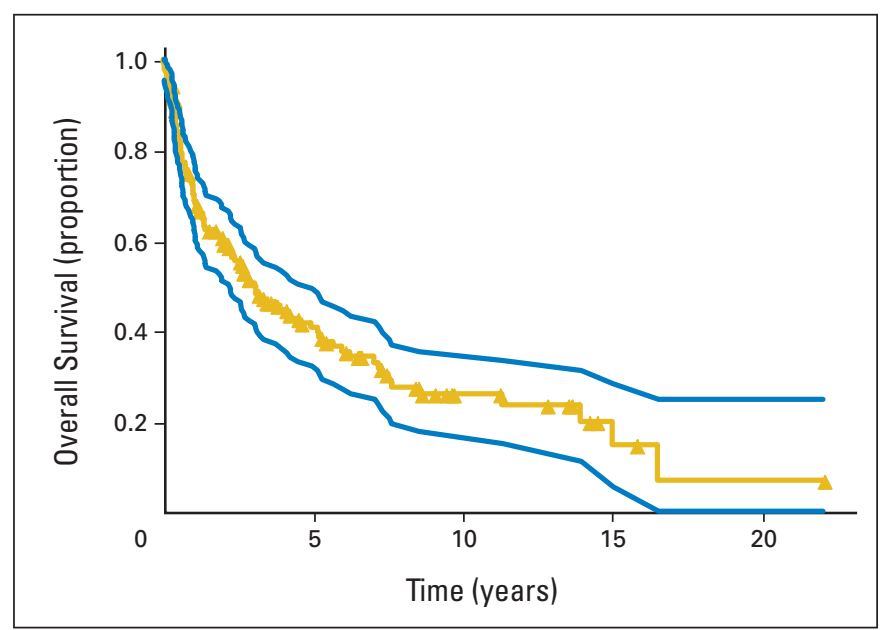

Fig 2. Overall survival (yellow line; median, 3.1 years; $95 \% \mathrm{Cl}, 2.2$ to 5.0 years) from date of first intra-arterial/blood-brain barrier disruption treatment (149 patients, 96 deaths) with $95 \% \mathrm{Cl}$ (blue lines). Symbols on lines indicate censored observations. 
resection $(P=.72$, Wilcoxon test) were not significant predictors. Patients younger than 60 years $(n=78)$ had a median OS of 5.2 years and a 5 -year survival rate of $52 \%$; patients $\geq 60$ years of age had median OS of 2.2 years and a 5 -year survival rate of $30 \%(P<.0019)$. A plateau in OS was seen at 8.5 years in patients younger than 60 years, suggesting that some of these 13 patients may have achieved a "cure."

The best predictive model for OS included MSKCC class 3 (age $\geq 50$ years and KPS $<70$; hazard ratio $=9.08 ; P<.0001$ ), age $\geq 60$ years with KPS less than 70 (hazard ratio $=0.150 ; P<.0001$ ), and age older than 60 years (hazard ratio $=2.39 ; P=.0008$ ). To understand these interactions of age with KPS, all combinations of age younger than 50 , age 50 to 60 , and age $\geq 60$ years and KPS less than 70 and $\geq 70$ were fit in one model. The difference among these six combinations was highly significant $(P<.0001)$. Visual inspection of the survival curves and improvement in model fit (based on Akaike's information criterion) suggested three risk groups: low risk (age $<60$ years and KPS $\geq 70$ ), moderate risk (age $<50$ years with KPS $<70$ or age $\geq 60$ years), and high risk (age 50 to 60 years and KPS $<70$; Fig 3A). Estimated hazard ratios were 3.24 (95\% CI, 1.92 to 5.47) and 11.63 (95\% CI, 5.33 to 25.37 ) for the moderate- and high-risk groups, respectively. Median OS for these three risk groups were 13.9 years (95\% CI, 5.2 years to not reached) for the low risk group, 2.3 years (95\% CI, 1.3 to 3.1 years) for the moderate risk group, and 0.6 years ( $95 \%$ CI, 0.3 to 0.9 years) for the high-risk group. Similar analyses for PFS also identified three risk categories (albeit with different groups): low (age 50 to 60 years with KPS $\geq 70$ or age $<50$ years), moderate (age $\geq 60$ years), and high (age 50 to 60 years with KPS $<70$; Fig 3B). Estimated hazard ratios were 1.91 (95\% CI, 1.19 to 3.06$)$ and 10.01 (95\% CI, 4.61 to 21.75 ) for moderate and high risk, respectively.

When separately analyzed, the off-protocol patients $(n=18)$ had lower KPS and were older than the on-protocol patients $(\mathrm{n}=131)$. The 18 off-protocol patients had higher mean MSKCC risk scores, shorter median OS ( 1.02 years, 95\% CI, 0.37 to 3.75 ; $v 3.24$ years, $95 \%$ CI, 2.28 to 5.29 ), shorter median PFS ( 0.62 years, $95 \%$ CI, 0.18 to 1.99 ; $v 2.23$ years, $95 \%$ CI, 1.43 to 3.45 ), and a lower rate of CR than the on-protocol patients.

Eighty-seven (90.6\%) of the 96 deaths occurred more than 30 days after BBBD/IA. Forty-six patients (47.9\%) died as a result of CNS lymphoma progression. Systemic recurrence was the cause of death in nine patients $(9.4 \%)$. Three patients $(3.1 \%)$ died as a result of other toxicity such as respiratory complications, five patients (5.2\%) died as a result of CNS toxicity, seven patients (7.3\%) died as a result of other causes such as cardiac disease, and in 17 patients (17.7\%), the cause of death was unknown.

Nine $(9.4 \%)$ of the 96 deaths occurred less than 30 days after BBBD/IA. One patient died within 48 hours; autopsy showed a pulmonary embolism. Eight patients died 3 to 30 days after BBBD/IA. Four of these patients died of infection, and four patients died from complications related to carotid dissection, myocardial infarction, heart failure, and unknown cause (one each).

\section{Treatment-Related Complications}

A total of 697 complications were identified during 2,079 BBBD procedures (33.5\%). The complications are summarized in Table 3. The most frequent complication was periprocedural focal seizures occurring in 50 patients (33.6\%; $9.2 \%$ of procedures). The majority of these occurred during barrier disruption and chemotherapy infusion, were aborted with IV barbiturates, and resulted in no permanent

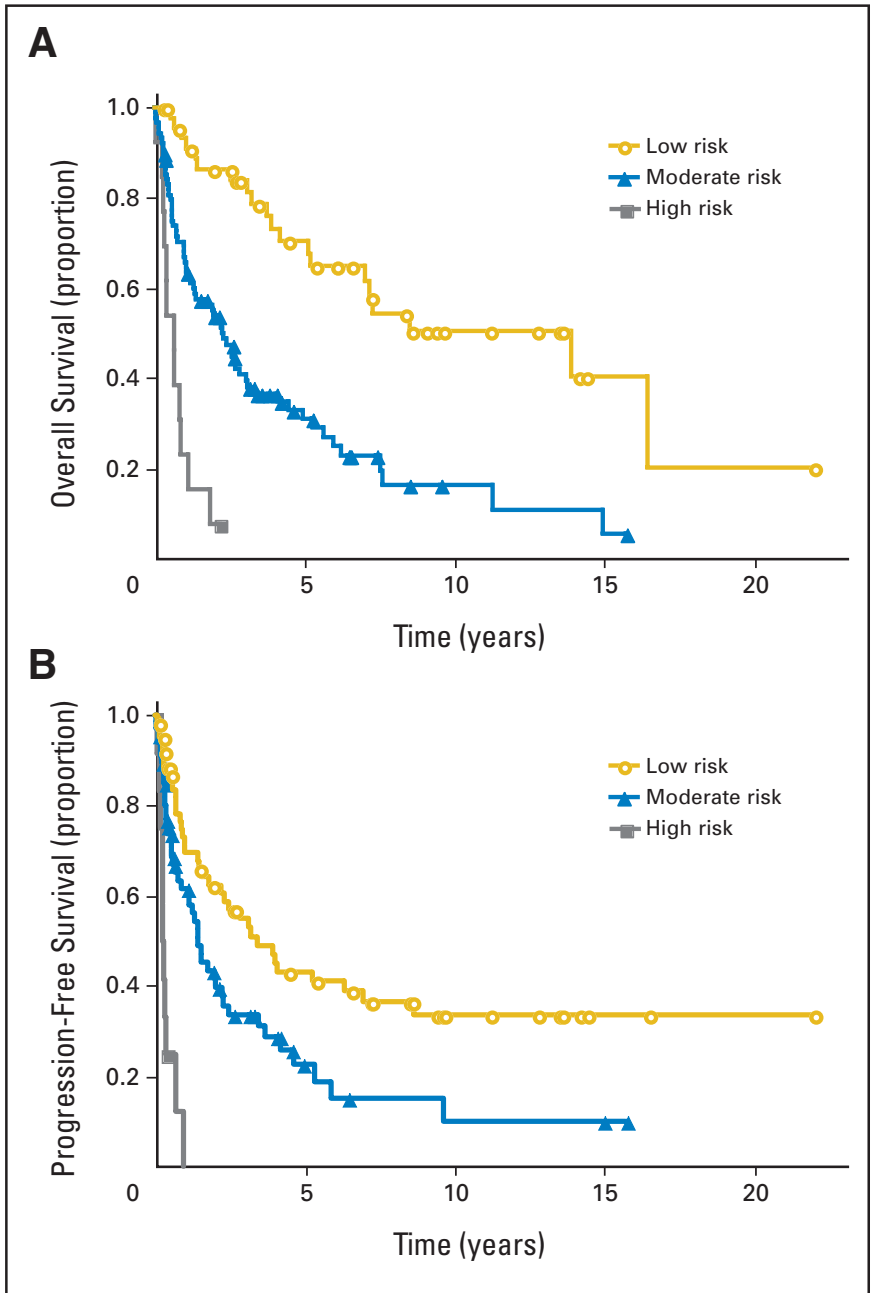

Fig 3. (A) Overall survival according to proposed risk groups. Survival from date of first intra-arterial/blood-brain barrier disruption treatment stratified by age and Karnofsky performance score (KPS). Low risk, age younger than 60 years with KPS $\geq 70$ (47 patients); moderate risk, age older than 60 years with any KPS or age younger than 50 years with KPS less than 70 (89 patients); and high risk, age 50 to less than 60 years with KPS less than 70 (13 patients). Symbols on lines indicate censored observations. (B) Progression-free survival according to proposed risk groups. Progression-free survival from date of first intra-arterial/bloodbrain barrier disruption treatment stratified by age and KPS. Low risk, age 50 to less than 60 years with KPS greater than 70, or age less than 50 years with any KPS (65 patients); moderate risk, age $\geq 60$ years with any KPS (71 patients); and high risk, age 50 to less than 60 years with KPS less than 70 (13 patients). Symbols on lines indicate censored observations.

neurologic dysfunction or uncontrolled seizures. Patients who experienced seizures had an increased likelihood to seize during subsequent procedures (191 seizures occurred in 50 patients; median of three seizures per patient). The overall rate of procedural morbidity is 327 events (15.7\% of procedures). However, if focal seizures are excluded, ${ }^{29}$ there were $136(6.5 \%)$ of 2,079 events. Clinical strokes occurred in 11 patients (7.4\%); four patients were left with permanent neurologic deficits. The estimated risk of permanent neurologic deficit is $0.2 \%$ per IA/BBBD procedure.

Chemotherapy toxicity and underlying malignant conditionrelated complications accounted for 370 events (17.8\%). Hematologic abnormalities were the most common presentation: RBC transfusions (3.6\% of procedures) and granulocytopenic fever $(2.8 \%)$. These patients had a hypercoagulable tendency with a $2.6 \%$ incidence of deep 


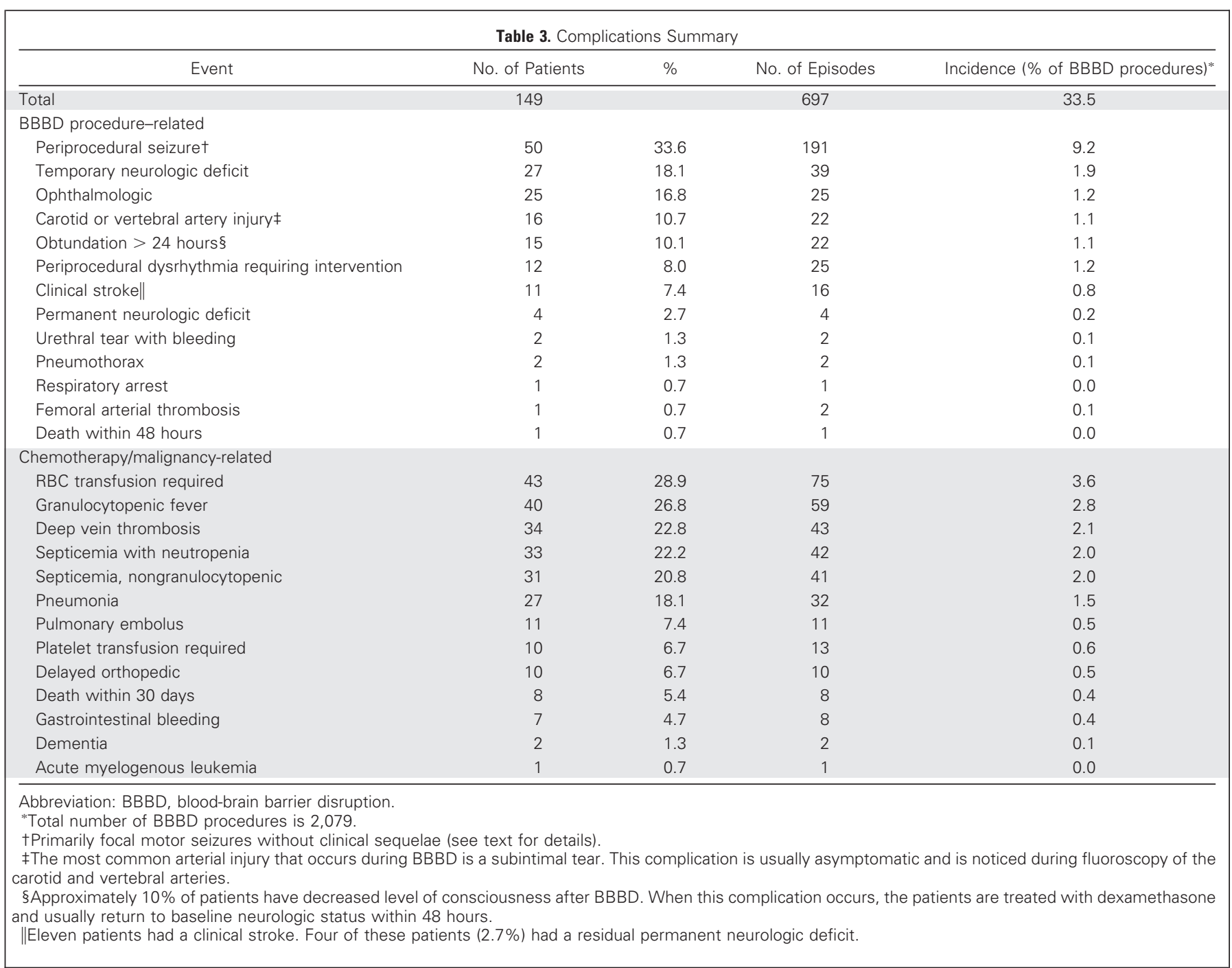

vein thrombosis or pulmonary embolism periprocedurally. Twentyfour patients (16.1\%) discontinued the 12-month regimen because of complications; 11 patients discontinued treatment because of secondary infections.

\section{DISCUSSION}

Data from three multicenter PCNSL trials that used chemotherapy (high-dose IV methotrexate) alone (New Approaches to Brain Tumor Therapy, ${ }^{30}$ German Cancer Society Neuro-Oncology Working Group (NOA) $03,{ }^{31}$ and European Organisation for Research and Treatment of Cancer [EORTC] 26952, ${ }^{32}$ ) are summarized in Table 4. Our results with IA methotrexate/BBBD demonstrated comparable if not superior outcomes to high-dose IV methotrexate used in the other three multicenter trials.

Similarly, the results of Radiation Therapy Oncology Group 93$10^{33}$ and EORTC $20962^{34}$ are summarized in Table 4. Both of these trials used combination chemotherapy and brain irradiation. IA methotrexate-based BBBD achieved similar results without the neurocognitive sequelae associated with brain irradiation. ${ }^{35}$ Caution must be exercised, as definitive conclusions cannot be drawn when comparing our results with those of other multicenter nonrandomized clinical trials of newly diagnosed patients with PCNSL. Nonetheless, we feel that summarizing results obtained from the other multicenter studies, as we have done in Table 4, may be of interest to clinicians. Given the rarity of PCNSL and the limited number of institutions currently trained in $\mathrm{BBBD}$, a randomized PCNSL trial using $\mathrm{BBBD}$ as one arm is currently not possible.

Cognitive outcomes were not available for all patients in our series; however, a subset of these patients have been evaluated as part of other published series. ${ }^{11,21,22,35-39}$ Neuropsychologic assessments include a comprehensive test battery conducted by neuropsychologists. Results revealed cognitive improvement or preservation in the majority of patients relative to pretreatment status at follow-up, between 1 to 7 years after achieving CR. ${ }^{11,21,22,35-39}$ Further evaluation of neurocognitive outcomes in long-term survivors is underway ( $\mathrm{L}$. Maron, personal communication, July 2008).

Toxicity in BBBD-treated patients is generally manageable. However, the treatment delivery regimen is complex and should be undertaken only by trained teams at centers where neuro-oncology, 


\begin{tabular}{|c|c|c|c|c|c|c|}
\hline \multirow[b]{2}{*}{ Study } & \multirow[b]{2}{*}{ No. of Patients } & \multirow[b]{2}{*}{$\mathrm{RR}(\mathrm{CR}+\mathrm{PR} ; \%)$} & \multicolumn{2}{|c|}{ PFS (months) } & \multicolumn{2}{|c|}{ OS (months) } \\
\hline & & & Median & $95 \% \mathrm{Cl}$ & Median & $95 \% \mathrm{Cl}$ \\
\hline NABTT $96-07,30$ high-dose methotrexate & 25 & 74 & 12.8 & & Not reached (>23) & \\
\hline NOA- $03,{ }^{31}$ high-dose methotrexate & 37 & 35 & 10 & & 25 & \\
\hline EORTC $26952,{ }^{32}$ high-dose methotrexate & 50 (> 60 years old $)$ & 48 & 6.8 & 3.4 to 10.6 & 14.3 & 6.2 to 42 \\
\hline RTOG $93-10,{ }^{33}$ high-dose methotrexate plus WBRT & 102 & 94 & 24 & & 36.9 & \\
\hline EORTC $20962,{ }^{34}$ high-dose methotrexate plus WBRT & $52(<65$ years old $)$ & 81 & - & & 46 & \\
\hline \multicolumn{7}{|l|}{ BBBD consortium, current study } \\
\hline Total patients & 149 & 81.9 & 21.3 & 15.1 to 34.0 & 37.1 & 27.0 to 59.5 \\
\hline Age $<60$ years & 78 & 83.3 & 29.8 & 12.2 to 49.1 & 61.9 & 34.2 to 167.3 \\
\hline Age $\geq 60$ years & 71 & 80.3 & 17.6 & 10.6 to 27.6 & 26.6 & 14.5 to 37.1 \\
\hline
\end{tabular}

interventional neurosurgery/neuroradiology, neuroanesthesia, and experienced oncology nursing are available.

Potential predictors of OS and PFS are combinations of age and KPS. Other potential predictors were not statistically significant when added to these models, and the hazard ratios for the moderate and high-risk groups were not changed when these other potential predictors were added. We also assessed MSKCC risk classes, but not the International Extranodal Lymphoma Study Group score (serum lactate dehydrogenase and CSF protein were not regularly available). ${ }^{40}$ Comparisons of multivariable models suggested that, in this case series, neither MSKCC risk categories nor age younger than 60 or $\geq 60$ years and KPS less than 70 or $\geq 70$ were adequate predictors. Six age-KPS combinations were fit and then grouped into three risk categories. The high-risk group (age 50 to 60 years and KPS $<70$ ) was the likely reason for this interaction. This high-risk group included only 13 patients and may reflect random, small subgroup variation (albeit a group with markedly poor OS and PFS). Many of these 13 patients had numerous premorbid conditions or concomitant complications unrelated to treatment (eg, one patient died secondary to spinal cord injury complications after a fall). Drawing any specific conclusions about this small subset of patients is risky and may be unnecessary. These risk groups are not intended to represent general risk categories (such as MSKCC or International Extranodal Lymphoma Study Group) but merely describe outcomes in this series.

Another potential confounder in this series is the small number of patients treated off-protocol at participating institutions. If we restrict the series to only on-protocol patients, the median OS increases slightly from the full series (from 3.1 to 3.2 years) and the median PFS increases (from 1.8 to 2.2 years). Even though the inclusion of off-protocol patients negatively impacts the efficacy reported in this series, we feel that their inclusion is important and more accurately reflects the overall therapeutic results using BBBD in patients with PCNSL.

Currently, systemic diffuse large B-cell lymphoma is treated with chemotherapy in conjunction with rituximab; however, this monoclonal antibody does not routinely cross the BBB and reach malignant lymphoma cells in the CNS. ${ }^{41-44} \mathrm{~A}$ reasonable approach might be to use a technique such as osmotic BBBD to deliver effective monoclonal antibody/chemotherapy or other novel agents.

In conclusion, we report response rates and OS from a large, multicenter series of newly diagnosed patients with PCNSL treated with IA methotrexate-based chemotherapy with osmotic BBBD, which confirm our previously reported single-center results. ${ }^{21,22}$ This treatment option resulted in durable tumor control, manageable toxicity, and the potential for deferral of radiation and its associated cognitive compromise. ${ }^{11,22,45}$ BBBD results in enhanced delivery not simply to areas with overtly leaky BBB associated with enhancing tumor, but also to brain and CSF globally by as much as 50- to 100fold. ${ }^{46}$ The long patient follow-up suggests this is an effective first-line treatment option with a meaningful impact on OS and PFS, as well as neurocognitive status. ${ }^{35}$

\section{AUTHORS' DISCLOSURES OF POTENTIAL CONFLICTS} OF INTEREST

The author(s) indicated no potential conflicts of interest.

\section{AUTHOR CONTRIBUTIONS}

Conception and design: Lilyana Angelov, Nancy D. Doolittle, Dale F. Kraemer, Tali Siegal, Gene H. Barnett, David M. Peereboom, Glen Stevens, John McGregor, Kristoph Jahnke, Susan Bell, Edward A. Neuwelt Financial support: Edward A. Neuwelt

Administrative support: Lilyana Angelov, Edward A. Neuwelt Provision of study materials or patients: Lilyana Angelov, Tali Siegal, Gene H. Barnett, David M. Peereboom, Glen Stevens, John McGregor, Kristoph Jahnke, Cynthia A. Lacy, Edna Shalom, Sandra Ference, Susan Bell, Lisa Sorenson, Rose Marie Tyson, Marianne Haluska,

Edward A. Neuwelt

Collection and assembly of data: Lilyana Angelov, Nancy D. Doolittle, Kristoph Jahnke, Cynthia A. Lacy, Nancy A. Hedrick, Edna Shalom, Sandra Ference, Susan Bell, Lisa Sorenson, Rose Marie Tyson, Marianne Haluska

Data analysis and interpretation: Lilyana Angelov, Nancy D. Doolittle, Dale F. Kraemer, Cynthia A. Lacy, Edward A. Neuwelt

Manuscript writing: Lilyana Angelov, Nancy D. Doolittle, Dale F. Kraemer, Edward A. Neuwelt

Final approval of manuscript: Lilyana Angelov, Nancy D. Doolittle, Dale F. Kraemer, Tali Siegal, Gene H. Barnett, David M. Peereboom, Glen Stevens, John McGregor, Kristoph Jahnke, Cynthia A. Lacy, Nancy A. Hedrick, Edna Shalom, Sandra Ference, Susan Bell, Lisa Sorenson, Rose Marie Tyson, Marianne Haluska, Edward A. Neuwelt 


\section{REFERENCES}

1. Panageas KS, Elkin EB, DeAngelis $L M$, et al: Trends in survival from primary central nervous system lymphoma, 1975-1999: A population-based analysis. Cancer 104:2466-2472, 2005

2. Muldoon LL, Soussain C, Jahnke K, et al: Chemotherapy delivery issues in central nervous system malignancy: A reality check. J Clin Oncol 25:2295-2305, 2007

3. Neuwelt E, Abbott NJ, Abrey L, et al: Strategies to advance translational research into brain barriers. Lancet Neurol 7:84-96, 2008

4. Abrey LE, DeAngelis LM, Yahalom J: Longterm survival in primary CNS lymphoma. J Clin Oncol 16:859-863, 1998

5. Abrey LE, Yahalom J, DeAngelis LM: Treatment for primary CNS lymphoma: The next step. J Clin Oncol 18:3144-3150, 2000

6. DeAngelis LM, Yahalom J, Thaler HT, et al: Combined modality therapy for primary CNS lymphoma. J Clin Oncol 10:635-643, 1992

7. Glass J, Gruber ML, Cher L, et al: Preirradiation methotrexate chemotherapy of primary central nervous system lymphoma: Long-term outcome. J Neurosurg 81:188-195, 1994

8. Ferreri AJ, Abrey LE, Blay JY, et al: Summary statement on primary central nervous system lymphomas from the Eighth International Conference on Malignant Lymphoma, Lugano, Switzerland, June 12 to 15, 2002. J Clin Oncol 21:2407-2414, 2003

9. Blay JY, Conroy T, Chevreau C, et al: Highdose methotrexate for the treatment of primary cerebral lymphomas: Analysis of survival and late neurologic toxicity in a retrospective series. J Clin Oncol 16:864-871, 1998

10. Correa DD, DeAngelis LM, Shi W, et al: Cognitive functions in survivors of primary central nervous system lymphoma. Neurology 62:548-555, 2004

11. Dahlborg SA, Henner WD, Crossen JR, et al: Non-AIDS primary CNS lymphoma: First example of a durable response in a primary brain tumor using enhanced chemotherapy delivery without cognitive loss and without radiotherapy. Cancer J Sci Am 2:166-174, 1996

12. Harder $\mathrm{H}$, Holtel H, Bromberg JE, et al: Cognitive status and quality of life after treatment for primary CNS lymphoma. Neurology 62:544-547, 2004

13. Lai R, Abrey LE, Rosenblum MK, et al: Treatment-induced leukoencephalopathy in primary CNS Iymphoma: A clinical and autopsy study. Neurology 62:451-456, 2004

14. O'Brien P, Roos D, Pratt G, et al: Phase II multicenter study of brief single-agent methotrexate followed by irradiation in primary CNS lymphoma. $\mathrm{J}$ Clin Oncol 18:519-526, 2000

15. Omuro AM, Ben-Porat LS, Panageas KS, et al: Delayed neurotoxicity in primary central nervous system lymphoma. Arch Neurol 62:1595-1600, 2005

16. Omuro AM, DeAngelis LM, Yahalom J, et al: Chemoradiotherapy for primary CNS lymphoma: An intent-to-treat analysis with complete follow-up. Neurology 64:69-74, 2005

17. Pels H, Schmidt-Wolf IG, Glasmacher A, et al: Primary central nervous system lymphoma: Results of a pilot and phase II study of systemic and intraventricular chemotherapy with deferred radiotherapy. J Clin Oncol 21:4489-4495, 2003

18. Shah GD, Yahalom J, Correa DD, et al: Combined immunochemotherapy with reduced wholebrain radiotherapy for newly diagnosed primary CNS lymphoma. J Clin Oncol 25:4730-4735, 2007

19. Batchelor T, Loeffler JS: Primary CNS lymphoma. J Clin Oncol 24:1281-1288, 2006

20. Gavrilovic IT, Hormigo A, Yahalom J, et al: Long-term follow-up of high-dose methotrexatebased therapy with and without whole brain irradiation for newly diagnosed primary CNS Iymphoma. J Clin Oncol 24:4570-4574, 2006

21. McAllister LD, Doolittle ND, Guastadisegni PE, et al: Cognitive outcomes and long-term follow-up results after enhanced chemotherapy delivery for primary central nervous system lymphoma. Neurosurgery 46:51-60, 2000; discussion 60-61

22. Neuwelt EA, Goldman DL, Dahlborg SA, et al: Primary CNS lymphoma treated with osmotic blood-brain barrier disruption: Prolonged survival and preservation of cognitive function. J Clin Oncol 9:1580-1590, 1991

23. Doolittle ND, Miner ME, Hall WA, et al: Safety and efficacy of a multicenter study using intraarterial chemotherapy in conjunction with osmotic opening of the blood-brain barrier for the treatment of patients with malignant brain tumors. Cancer 88:637647, 2000

24. Jahnke K, Doolittle ND, Muldoon LL, et al: Implications of the blood-brain barrier in primary central nervous system lymphoma. Neurosurg Focus 21:E11, 2006

25. Smith JR, Rosenbaum JT, Wilson DJ, et al: Role of intravitreal methotrexate in the management of primary central nervous system lymphoma with ocular involvement. Ophthalmology 109:1709-1716, 2002

26. Cohen IJ: Defining the appropriate dosage of folinic acid after high-dose methotrexate for childhood acute lymphatic leukemia that will prevent neurotoxicity without rescuing malignant cells in the central nervous system. J Pediatr Hematol Oncol 26:156-163, 2004

27. Macdonald DR, Cascino TL, Schold SC Jr, et al: Response criteria for phase II studies of supratentorial malignant glioma. J Clin Oncol 8:12771280, 1990

28. Abrey LE, Ben-Porat $L$, Panageas KS, et al: Primary central nervous system lymphoma: The Memorial Sloan-Kettering Cancer Center prognostic model. J Clin Oncol 24:5711-5715, 2006

29. Marchi N, Angelov L, Masaryk T, et al: Seizure-promoting effect of blood-brain barrier disruption. Epilepsia 48:732-742, 2007

30. Batchelor T, Carson K, O'Neill A, et al: Treatment of primary CNS lymphoma with methotrexate and deferred radiotherapy: A report of NABTT 96-07. J Clin Oncol 21:1044-1049, 2003

31. Herrlinger $U$, Schabet $M$, Brugger $W$, et al: German Cancer Society Neuro-Oncology Working Group NOA-03 multicenter trial of single-agent highdose methotrexate for primary central nervous system lymphoma. Ann Neurol 51:247-252, 2002

32. Hoang-Xuan K, Taillandier L, Chinot $O$, et al: Chemotherapy alone as initial treatment for primary CNS lymphoma in patients older than 60 years: $\mathrm{A}$ multicenter phase II study (26952) of the European Organization for Research and Treatment of Cancer
Brain Tumor Group. J Clin Oncol 21:2726-2731, 2003

33. DeAngelis LM, Seiferheld W, Schold SC, et al: Combination chemotherapy and radiotherapy for primary central nervous system lymphoma: Radiation Therapy Oncology Group Study 93-10. J Clin Oncol 20:4643-4648, 2002

34. Poortmans PM, Kluin-Nelemans HC, HaaxmaReiche $\mathrm{H}$, etal: High-dose methotrexate-based chemotherapy followed by consolidating radiotherapy in non-AIDS-related primary central nervous system lymphoma: European Organization for Research and Treatment of Cancer Lymphoma Group Phase II Trial 20962. J Clin Oncol 21:4483-4488, 2003

35. Correa DD, Maron L, Harder H, et al: Cognitive functions in primary central nervous system lymphoma: Literature review and assessment guidelines. Ann Oncol 18:1145-1151, 2007

36. Crossen JR, Garwood D, Glatstein E, et al: Neurobehavioral sequelae of cranial irradiation in adults: A review of radiation-induced encephalopathy. J Clin Oncol 12:627-642, 1994

37. Crossen JR, Goldman DL, Dahlborg SA, et al: Neuropsychological assessment outcomes of nonacquired immunodeficiency syndrome patients with primary central nervous system lymphoma before and after blood-brain barrier disruption chemotherapy. Neurosurgery 30:23-29, 1992

38. Neuwelt EA, Guastadisegni $P E$, Varallyay $P$, et al: Imaging changes and cognitive outcome in primary CNS lymphoma after enhanced chemotherapy delivery. AJNR Am J Neuroradiol 26:258-265, 2005

39. Roman-Goldstein S, Mitchell P, Crossen JR, et al: MR and cognitive testing of patients undergoing osmotic blood-brain barrier disruption with intraarterial chemotherapy. AJNR Am J Neuroradio 16:543-553, 1995

40. Ferreri AJ, Blay JY, Reni M, et al: Prognostic scoring system for primary CNS lymphomas: The International Extranodal Lymphoma Study Group experience. J Clin Oncol 21:266-272, 2003

41. Rubenstein $\mathrm{JL}$, Combs $\mathrm{D}$, Rosenberg J, et al: Rituximab therapy for CNS lymphomas: Targeting the leptomeningeal compartment. Blood 101:466468,2003

42. Feugier $\mathrm{P}$, Virion JM, Tilly $H$, et al: Incidence and risk factors for central nervous system occurrence in elderly patients with diffuse large-B-cell lymphoma: Influence of rituximab. Ann Oncol 15: 129-133, 2004

43. Doolittle ND, Abrey LE, Shenkier TN, et al: Brain parenchyma involvement as isolated central nervous system relapse of systemic non-Hodgkin lymphoma: An International Primary CNS Lymphoma Collaborative Group report. Blood 111:10851093, 2008

44. Doolittle ND, Jahnke $K$, Belanger $R$, et al: Potential of chemo-immunotherapy and radioimmunotherapy in relapsed primary central nervous system (CNS) lymphoma. Leuk Lymphoma 48: 1712-1720, 2007

45. Tyson RM, Siegal T, Doolittle ND, et al: Current status and future of relapsed primary central nervous system lymphoma (PCNSL). Leuk Lymphoma 44:627-633, 2003

46. Neuwelt EA, Diehl JT, Vu LH, et al: Monitoring of methotrexate delivery in patients with malignant brain tumors after osmotic blood-brain barrier disruption. Ann Intern Med 94:449-454, 1981 